\title{
Numerical shape-from-shading revisited
}

\author{
Hiroaki Santo*, Masaki Samejima and Yasuyuki Matsushita
}

\begin{abstract}
This paper revisits the numerical shape-from-shading method proposed in early 1980s. The original problem is non-convex due to the unit norm constraint for surface normal, and the existing approaches including the original Ikeuchi and Horn's work uses approximate solution strategies for the original problem. This paper instead studies relaxation strategies for the original non-convex constraint and describes corresponding solution techniques that are built upon advanced convex optimization. We analyze the effect of the relaxations in terms of resulting accuracy and computational complexity.
\end{abstract}

Keywords: Shape-from-shading, Convex optimization

\section{Introduction}

Shape-from-shading [11] is a problem of determining shape in the form of surface normal from the shading distribution observed in a single image. While a human can naturally achieve this task, it is computationally nontrivial and still remains as one of the central problems in computer vision.

The major difficulty arises from the fact that the problem is under-constrained, i.e., there are many solutions that satisfy the image formation model. In other words, there exists a set of shapes that yields exactly the same shading appearance under a fixed lighting condition. To overcome this issue, previous approaches incorporate additional priors, such as the smoothness constraint [13]. With such priors, it has been shown that the shape-fromshading problem can be better constrained.

There is another difficulty in shape-from-shading that is often overlooked, the non-convex nature of the problem due to the unit norm constraint. Even assuming a linear (Lambertian) reflectance model, the problem of inferring shape in the form of surface normal requires the surface normal vector to be in the unit norm, namely, $\|\mathbf{n}\|_{2}=1$, for a surface normal vector $\mathbf{n} \in \mathbb{R}^{3}$. Oftentimes, a twoparameter notation of a surface normal vector $(p, q, 1)^{\top}$ is used, but it comes with the normalization of its magnitude, resulting in $\mathbf{n}=(p, q, 1)^{\top} / \sqrt{p^{2}+q^{2}+1}$. It makes the unit norm constraint somehow implicit;

*Correspondence: santo.hiroaki@ist.osaka-u.ac.jp

Graduate School of Information Science and Technology, Osaka University, Osaka, Japan however, the problem is fundamentally unchanged and the non-convexity of the problem still remains ${ }^{1}$.

This paper studies the effect of the unit norm constraint $\left(\|\mathbf{n}\|_{2}=1\right)$ that always appears in shape-fromshading problems, under the conventional assumptions of orthographic projection and calibrated point light source. This constraint makes the overall problem non-convex; therefore, it is important to understand its property and develop work-around if any for the method to be applied in practical situations. We illustrate various relaxation strategies and corresponding solution methods and assess the effect of the approximations. Our study puts its basis on the early work of numerical shape-from-shading [13] and revisits the problem with advanced convex relaxation and optimization methods that have been more recently developed.

\subsection{Related works}

Since Horn's original work [11], the problem of shapefrom-shading has been one of the central problems in computer vision. While the shape-from-shading problem can be described in a simple manner, it exhibits a mathematically rich structure. There have been a numerous number of previous works that study shape-fromshading, and an excellent survey of the early methods is found in [45]. The survey categorizes the approaches into four classes: minimization [5, 13], propagation [11, 19], basis representation [23, 28], and linear approximation $[27,37]$ approaches. Our method falls in the class of minimization approaches, in which the smoothness of the surface normal is maximized under some constraints. The 
vast majority of the early works focuses on the solution strategies; however, surprisingly very few works explicitly discussed the issue of the non-convex nature of the problem until more recently $[7,18]$. Early methods tried to avoid the issue of non-convexity by their customized solution technique. For example, Ikeuchi and Horn [13] iterate between solving the problem without the nonconvex constraint and normalizing the surface normal. Szeliski's work [35] has used a gradient-descent method for obtaining the surface normal in conjunction with a hierarchical basis representation based on scale-space theory [38] for shape and its gradient, effectively avoiding local minimas. More recently, Xiong et al. [43] used a locally quadratic shape representation for robust inference of the global shape. A newer survey of shape-fromshading [6] provided a comprehensive summary of recent shape-from-shading methods.

Most of the existing methods, including the original shape-from-shading [11] and our method, assume an orthographic camera projection and calibrated light condition, i.e., the light source direction is known. Recently, methods to alleviate with these restrictions have been proposed. Tankus et al. [36] proposed a shape-from-shading method under a perspective projection based on an extension of fast marching [20]. They have evaluated their method using synthetic images and the medical images recorded by an endoscopy and demonstrated improvement in accuracy by the perspective projection model. Richter et al. [30] used a learning-based approach for estimating surface normal under perspective and uncalibrated conditions. Their method uses a regression forest for determining surface normal trained with synthetic data and has shown promising results.

While most of the methods assume a point light source, Queau et al. [29] proposed a shape-from-shading method under natural illumination. They used a variational method for ensuring smoothness of surface normal through regularization by solving partial differential equations. Their method demonstrates robustness in estimation without tedious tuning of a regularization parameter.

For the purpose of making shape-from-shading applicable to real-world scenarios, there are threads of works that aim at relaxation of restrictive assumptions. They include the relaxations of known and uniform albedo assumption [2] using a coarse depth information, spatially uniform illumination assumption [8], and known illumination assumption [31] with a discriminative learning approach. With these advancements, shape-fromshading has been successfully applied to some real-world applications, such as endoscopy [42], recovery of shape with high-frequency details [41, 44], and face recognition $[3,34]$ to list a few. Our study also aims at broadening the use of shape-from-shading, and this paper particularly studies the unit norm constraint that is inherent in shapefrom-shading problems. Unlike previous approaches that introduce new assumptions for making the problem more tractable, our focus is to analyze the behavior of the unit norm constraint and its relaxed surrogates.

\section{Background}

Given a measurement vector $\mathbf{m} \in \mathbb{R}^{p}$ that consists of $p$ pixel observations under a distant light $\mathbf{l} \in \mathbb{R}^{3},\|\mathbf{l}\|_{2}=1$, we wish to recover the surface normal map (scaled by albedo) $\mathbf{N} \in \mathbb{R}^{3 \times p}$ based on the Lambertian image formation model

$$
\mathbf{m}^{\top}=\mathbf{l}^{\top} \mathbf{N} .
$$

We revisit the original numerical shape-from-shading formulation [13] using a matrix notation because of its simplicity of notations. The three constraints introduced in the original work [13] — brightness, smoothness, and occluding boundary constraints-can be written as follows:

Brightness constraint. The brightness constraint ensures the agreement among observations $\mathbf{m}$, lighting $\mathbf{l}$, and surface normal $\mathbf{N}$ via the Lambertian image formation model:

$$
\mathbf{l}^{\top} \mathbf{N}-\mathbf{m}^{\top} \rightarrow \mathbf{0}
$$

Smoothness constraint. Smoothness constraint ensures the surface normal estimates have locally smooth variations. Using a 2D Laplacian matrix $\mathbf{D} \in \mathbb{R}^{p \times p}$ defined over grid locations in a valid image region, the smoothness constraint can be written as

$$
\text { ND } \rightarrow \mathbf{0 .}
$$

Occluding boundary constraint. At pixels on an occluding boundary, it is assumed that the surface orientation information is available. Namely, it assumes that the surface normal direction is perpendicular to the tangent line of the object boundary, looking outward. Let $\mathbf{F}$, a diagonal $p \times p$ matrix, indicate the pixel locations where the occluding boundary constraint is applicable ( 1 for such pixels and 0 otherwise), and a matrix $\mathbf{G} \in \mathbb{R}^{3 \times p}$ contains the corresponding surface normal information. For example, if the $i$ th pixel is at the occluding boundary, $F_{i, i}=1$ and $\mathbf{g}_{i}=\mathbf{n}_{i}$, where $\mathbf{g}_{i}$ and $\mathbf{n}_{i}$ correspond to the $i$ th column vectors of $\mathbf{G}$ and $\mathbf{N}$, respectively. For non-occluding boundary pixels, $F_{j, j}=0$ and $\mathbf{g}_{j}=\mathbf{0}$. With these notations, the occluding boundary constraint can be written as

$$
\mathbf{N F}-\mathbf{G} \rightarrow \mathbf{0} .
$$

Unit norm constraint. Another important constraint is a unit norm constraint for surface normal vectors. Namely, 
the norm of a surface normal vector $\mathbf{n}_{i} \in \mathbb{R}^{3}$, corresponding to a column vector of $\mathbf{N}\left(=\left[\mathbf{n}_{1}, \ldots, \mathbf{n}_{p}\right]\right)$ needs to satisfy

$$
\left\|\mathbf{n}_{i}\right\|_{2}=1, \quad \forall i \in\{1, \ldots, p\} .
$$

In addition, we are interested in surface normals that are visible from a camera; thus, an additional constraint $0 \leq$ $n_{z}$ can be placed.

In the original formulation [13], the smoothness constraint (3) is regarded as an objective function to minimize while the rest are treated as hard constraints as

$$
\begin{array}{cl}
\underset{\mathbf{N}}{\operatorname{minimize}} & \frac{1}{2}\|\mathbf{N D}\|_{F}^{2} \\
\text { subject to } & \mathbf{l}^{\top} \mathbf{N}-\mathbf{m}^{\top}=0, \mathbf{N F}-\mathbf{G}=\mathbf{0}, \\
& \left\|\mathbf{n}_{i}\right\|_{2}^{2}=1,0 \leq n_{i z}, \quad \forall i \in\{1 \ldots p\} .
\end{array}
$$

This problem is a non-convex QCQP (quadratically constrained quadratic program) due to the non-convex constraint $\left\|\mathbf{n}_{i}\right\|_{2}^{2}=1$ and understood as a NP-hard problem. In other words, the computational difficulty arises solely due to the unit norm constraint $\left\|\mathbf{n}_{i}\right\|_{2}^{2}=1$. The original paper [13] tackled the problem essentially by iteratively solving a relaxed subproblem without the norm constraint. This paper revisits this problem and studies possible relaxations of the norm constraint and their effects.

\section{Relaxations and solution methods}

In the original formulation (6), the unit norm constraint for surface normal is a non-convex quadratic equality, which is the source of the non-convexity of the overall problem. This section describes convex relaxation strategies for shape-from-shading and their solution methods. We consider the following three types of convex relaxations in addition to the original non-convex problem:

$\begin{array}{ll}\text { ORIGINAL (non-convex) } & \left\|\mathbf{n}_{i}\right\|_{2}^{2}=1, \quad 0 \leq n_{i z} \\ \text { INSIDE } & \left\|\mathbf{n}_{i}\right\|_{2}^{2} \leq 1, \quad 0 \leq n_{i z} \\ \text { BOX } & -1 \leq n_{i x}, \quad n_{i y} \leq 1, \quad 0 \leq n_{i z} \\ \text { OPEN } & 0 \leq n_{i z}\end{array}$

Figure 1 shows feasible regions of the original unit norm constraint and the relaxed constraints. The "ORIGINAL" constraint says that the norm of surface normal must be on the hemisphere formed by $\left\|\mathbf{n}_{i}\right\|_{2}^{2}=1$ and $n_{z} \geq 0$. The "INSIDE" relaxation is a convex surrogate for the unit norm constraint, turning the original constraint into a quadratic inequality constraint. The "BOX" relaxation uses a looser convex approximation to the original constraint to form linear inequality constraints that correspond to ranges of each elements of surface normal. Finally, the "OPEN" relaxation fully removes the unit norm constraint and allows solutions anywhere in the half-space $n_{z} \geq 0$. Aside from the ORIGINAL constraint, the three relaxed constraints are all convex, and thus, they turn the whole problem into convex. In what follows, we discuss solution methods for these settings.

\subsection{ORIGINAL constraint}

Because the feasible region of the original unit norm constraint is non-convex, deriving its exact solution is generally difficult. To make it computationally tractable, the original problem can be approximated to either Lagrangian relaxation or semidefinite programming (SDP) relaxation [1, 7]. In general, the SDP relaxation, which becomes a convex problem, better approximates the original problem unless the weighting factors for Lagrangian relaxation is carefully chosen and yields higher accuracy. However, linearization in SDP relaxation generates a huge dense matrix $\operatorname{vec}(\mathbf{N})^{\text {vec }}(\mathbf{N})\left(\in \mathbb{R}^{3 p \times 3 p}\right)$, which prohibits the method to work only with small images as pointed out in [7]. We now discuss the Lagrangian relaxation of the original problem (6) with weight parameters $\lambda_{1}, \lambda_{2}$, and $\lambda_{3}$ :

$$
\begin{array}{ll}
\underset{\mathbf{N}}{\operatorname{minimize}} & \frac{1}{2}\|\mathbf{N D}\|_{F}^{2}+\lambda_{1}\left\|\mathbf{I}^{\top} \mathbf{N}-\mathbf{m}^{\top}\right\|_{2}^{2} \\
& +\lambda_{2}\|\mathbf{N F}-\mathbf{G}\|_{F}^{2}+\lambda_{3} \sum_{i \in\{1 \ldots p\}}\left(\left\|\mathbf{n}_{i}\right\|_{2}^{2}-1\right)^{2} \\
\text { subject to } & 0 \leq n_{i z} .
\end{array}
$$

For convenience of later discussion, we vectorize $\mathbf{N}$ as $\mathbf{x}=\operatorname{vec}(\mathbf{N})=\left[\mathbf{n}_{1}^{\top}, \ldots, \mathbf{n}_{p}^{\top}\right]^{\top}$ and reformulate the problem (7) as:

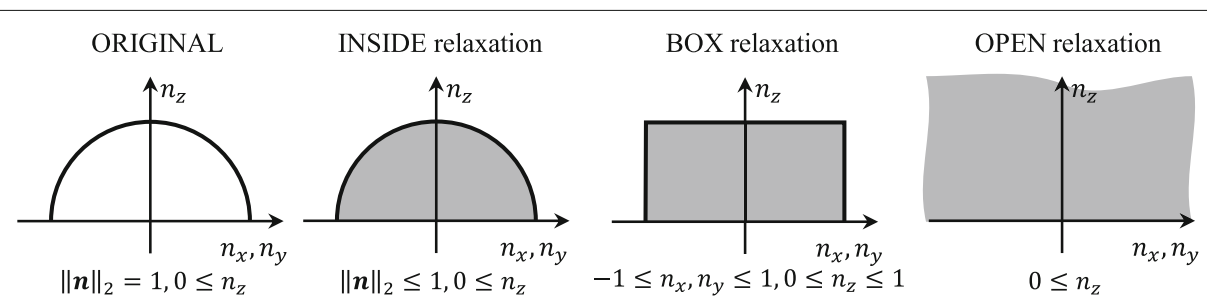

Fig. 1 Feasible regions of the unit norm constraint (ORIGINAL) and its relaxations (INSIDE, BOX, and OPEN) 


$$
\begin{array}{ll}
\underset{\mathbf{x}}{\operatorname{minimize}} & \frac{1}{2}\left\|\mathbf{D}_{\otimes} \mathbf{x}\right\|_{2}^{2}+\lambda_{1}\left\|\mathbf{L}_{\otimes} \mathbf{x}-\mathbf{m}^{\top}\right\|_{2}^{2} \\
& +\lambda_{2}\left\|\mathbf{F}_{\otimes} \mathbf{x}-\mathbf{g}\right\|_{2}^{2}+\lambda_{3} \sum_{i \in\{1 \ldots p\}}\left(\left\|\mathbf{n}_{i}\right\|_{2}^{2}-1\right)^{2}
\end{array}
$$

subject to $0 \leq n_{i z}$,

where

$$
\left\{\begin{aligned}
\mathbf{L}_{\otimes} & =\mathbf{I}_{p} \otimes \mathbf{I}^{\top}\left(\in \mathbb{R}^{p \times 3 p}\right) \\
\mathbf{D}_{\otimes} & =\mathbf{D} \otimes \mathbf{I}_{3}\left(\in \mathbb{R}^{3 p \times 3 p}\right) \\
\mathbf{F}_{\otimes} & =\mathbf{F} \otimes \mathbf{I}_{3}\left(\in \mathbb{R}^{3 p \times 3 p}\right) \\
\mathbf{g} & =\operatorname{vec}(\mathbf{G}),
\end{aligned}\right.
$$

with $\otimes$ representing the Kronecker product operator and $\mathbf{I}_{3}$ being a $3 \times 3$ identity matrix.

While the problem (8) is a non-convex nonlinear leastsquares problem with boundary conditions $0 \leq n_{i z}$, we can apply a variant of Levenberg-Marquardt algorithm $[24,26]$ that is designed for (convex) constrained problems [16] to seek a local minima. The updating formula from $\mathbf{x}$ at iteration $k$ denoted by $\mathbf{x}^{(k)}$ to $\mathbf{x}^{(k+1)}$ is given as:

$$
\mathbf{x}^{(k+1)}=\mathbf{x}^{k}+\mathbf{d}^{k} .
$$

The parameter $\mathbf{d}^{(k)}\left(\in \mathbb{R}^{3 p}\right)$ indicates the search direction of Levenberg-Marquardt algorithm and is determined by solving the subproblem described in Appendix 1 .

Lagrangian relaxation yields a good approximate solution to the original problem when $\lambda_{1}, \lambda_{2}$, and $\lambda_{3}$ are available and if we could solve the problem by overcoming the non-convexity. However, due to the non-convexity, the Levenberg-Marquardt method (or any other convex optimization methods) may be trapped in local minima depending on the initial guess $\mathbf{x}_{0}$. In addition, the best choice of $\lambda_{1}, \lambda_{2}$, and $\lambda_{3}$ depends on the target image, and unfortunately, the ideal values are generally inaccessible.

\subsection{INSIDE relaxation}

The INSIDE relaxation of the problem is formulated as:

$$
\begin{array}{cl}
\underset{\mathbf{x}}{\operatorname{minimize}} & \frac{1}{2}\left\|\mathbf{D}_{\otimes} \mathbf{x}\right\|_{2}^{2} \\
\text { subject to } & \mathbf{L}_{\otimes} \mathbf{x}-\mathbf{m}^{\top}=\mathbf{0}, \\
& \mathbf{F}_{\otimes} \mathbf{x}-\mathbf{g}=\mathbf{0}, \\
& 0 \leq \mathbf{s}_{i}^{\top} \mathbf{x}, \quad \forall i \in\{3,6, \ldots, 3 p\}, \\
& \mathbf{x}^{\top} \mathbf{K}_{i} \mathbf{x} \leq 1, \quad \forall i \in\{1, \ldots, p\},
\end{array}
$$

where $\mathbf{s}_{i}\left(\in \mathbb{R}^{3 p}\right)$ are single-entry vectors with one in row $i$ and zero elsewhere, and $\mathbf{K}_{i}$ is a block diagonal matrix:

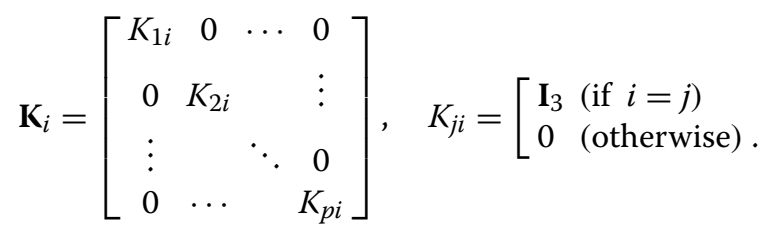

The relaxed problem is convex QCQP, which can be solved as a second-order cone program (SOCP) [25]. The details of the solution method are described in Appendix 2. While this SOCP problem can be solved more efficiently than the SDP relaxation to the original problem, it is still computationally demanding when the size of input image is large.

\subsection{BOX relaxation}

The Box relaxation problem, in which the unit norm constraint is replaced by range constraints of surface normal elements, can be written as:

$$
\begin{array}{cl}
\underset{\mathbf{x}}{\operatorname{minimize}} & \frac{1}{2}\left\|\mathbf{D}_{\otimes} \mathbf{x}\right\|_{2}^{2} \\
\text { subject to } & \mathbf{L}_{\otimes} \mathbf{x}-\mathbf{m}^{\top}=\mathbf{0}, \\
& \mathbf{F}_{\otimes} \mathbf{x}-\mathbf{g}=\mathbf{0}, \\
& 0 \leq \mathbf{s}_{j}^{\top} \mathbf{x} \leq 1, \quad \forall j \in\{3,6, \ldots 3 p\}, \\
& -1 \leq \mathbf{s}_{i}^{\top} \mathbf{x} \leq 1, \quad \forall i \in\{i \neq j\} .
\end{array}
$$

This problem is a linear constrained quadratic programming (LCQP) and also can be solved by the primal-dual interior point method [40]. Because the Karush-KuhnTucker (KKT) conditions for the BOX relaxation involve less quadratic terms than those for the INSIDE relaxation, the KKT equations for this case can be efficiently solved by a standard Newton's method (Appendix 3).

\subsection{OPEN relaxation}

The case for OPEN relaxation is rather straightforward. The problem in this case can be written in the form of LCQP as

$$
\begin{array}{ll}
\underset{\mathbf{x}}{\operatorname{minimize}} & \frac{1}{2}\left\|\mathbf{D}_{\otimes} \mathbf{x}\right\|_{2}^{2} \\
\text { subject to } & \mathbf{L}_{\otimes} \mathbf{x}-\mathbf{m}^{\top}=\mathbf{0}, \\
& \mathbf{F}_{\otimes} \mathbf{x}-\mathbf{g}=\mathbf{0}, \\
& 0 \leq \mathbf{s}_{i}^{\top} \mathbf{x}, \quad \forall i \in\{3,6, \ldots, 3 p\},
\end{array}
$$

and, again, it can be efficiently solved by a primal-dual interior point method [40].

\subsection{Piecewise solution method}

While the INSIDE relaxation approach shows higher accuracy than other relaxation strategies that are described above, its computational complexity rapidly grows along with the image size. Motivated by propagation approaches in shape-from-shading (see Section 2.2 of [45]), we develop an efficient piecewise solution strategy. 
The proposed method splits the image into small patches having some overlaps to the neighbors and estimates surface normal using the INSIDE relaxation starting from the most reliable patch. The reliability is determined by the number of the occluding boundary constraints in a patch; the more the constraints are provided, the better surface normal estimate is expected. Once the surface normal map $\hat{\mathbf{x}}$ for the most reliable patch is determined by the INSIDE relaxation method, the normal maps $\mathbf{x}$ of its neighbors are estimated by taking the surface normal estimates $\hat{\mathbf{x}}$ of the overlapped pixels as new constraints. Namely, the following additional constraint between $\hat{\mathbf{x}}$ and $\mathbf{x}$ is further enforced to the INSIDE relaxation setting:

$$
\mathbf{R}(\mathbf{x}-\hat{\mathbf{x}}) \rightarrow \mathbf{0},
$$

where $\mathbf{R}$ is a matrix that selects pixel locations where the surface normal estimates $\hat{\mathbf{x}}$ is available in the overlapped regions, i.e., $\mathbf{R}=\operatorname{diag}\left[r_{0}, \ldots, r_{p}\right]$, and $r_{i}=1$ if the pixel location $i$ has the estimated normal $\hat{\mathbf{x}}$ and $r_{i}=0$ otherwise. Since the surface normal estimates $\hat{\mathbf{x}}$ are subject to error, putting them as hard constraints has a chance of making the problem infeasible. Therefore, we treat the new constraints as a soft constraint with a positive weight parameter $\lambda$. The procedure for a target patch is written as

$$
\begin{array}{cl}
\underset{\mathbf{x}}{\operatorname{minimize}} & \frac{1}{2}\left\|\mathbf{D}_{\otimes} \mathbf{x}\right\|_{2}^{2}+\lambda \mathbf{R}(\mathbf{x}-\hat{\mathbf{x}}) \\
\text { subject to } & \mathbf{L}_{\otimes} \mathbf{x}-\mathbf{m}^{\top}=\mathbf{0}, \\
& \mathbf{F}_{\otimes} \mathbf{x}-\mathbf{g}=\mathbf{0}, \\
& 0 \leq \mathbf{s}_{i}^{\top} \mathbf{x}, \quad \forall i \in\{3,6, \ldots 3 p\}, \\
& \mathbf{x}^{\top} \mathbf{K}_{i} \mathbf{x} \leq 1, \quad \forall i \in\{1 \ldots p\} .
\end{array}
$$

Since solving the INSIDE relaxation setting by SOCP requires $O\left(n^{3}\right)$ computational complexity, where $n$ is the number of unknowns ( $3 p$ in our case), this patch splitting strategy makes the problem significantly more efficient at the cost of degradation of the accuracy. For example, when the patch size is set $1 / 10$ of the entire image size, it becomes 100 times faster $\left(1 / 10^{3}\right.$ computation is repeated 10 times).

As described, the solution method is sequential, i.e., if the initial estimate fails, the error may propagate to the rest of the estimation. However, by starting with the most reliable patch, this effect is alleviated, and in practice, we found the strategy is sufficiently reliable.

\section{Experiments}

This section shows experimental results using both synthetic and real-world images for the various settings for the unit norm constraint. The performance of the ORIGINAL problem and INSIDE, BOX, and OPEN relaxations are examined in terms of their accuracy and computation times. We also evaluate the effectiveness of the piecewise solution method described in Section 3.5. In addition, we compare these strategies with the original numerical shape-from-shading algorithm proposed by Ikeuchi and Horn [13] (labeled “ITERATIVE” hereafter), a polynomial shape-from-shading method proposed by Ecker and Jepson [7] (labeled "P-SFS"), and local shape prediction method proposed by Xiong et al. [43] (labeled "XIONG”).

For the ITERATIVE method [13], following the original method's procedure, we repeat the Newton step for the following problem for a few times (set to 5 in this evaluation based on our empirical test) starting from the initial guess $\mathbf{n}=(0,0,1)^{\top}$ :

$$
\begin{array}{ll}
\underset{\mathbf{N}}{\operatorname{minimize}} & \frac{1}{2}\|\mathbf{N D}\|_{F}^{2}+\lambda_{1}\left\|\mathbf{l}^{\top} \mathbf{N}-\mathbf{m}^{\top}\right\|_{2}^{2}+\lambda_{2}\|\mathbf{N F}-\mathbf{G}\|_{F}^{2} \\
\text { subject to } & 0 \leq n_{i z},
\end{array}
$$

and normalize the current estimate of the surface normal to $\left\|\mathbf{n}_{i}\right\|_{2}=1$. As such, it iteratively optimizes without the unit norm constraint, and during the iterations, it enforces the surface normal to have the unit norm by normalization. In the work of the P-SFS method [7], they propose an iterative procedure with exact line search, which is inherently non-convex, and its convex SDP relaxation. Since their method does not require boundary conditions, we align the setting to their setting and compare the performance with their SDP relaxation method. We use Gurobi Optimizer $^{2}$ as a solver for SDP problems. XIONG method [43] assumes the quadratic representation of local shape and infers the local shape for each small image patches separately. We use their implementation that is publicly available $^{3}$ and their default parameters for our experiment. In our all experiments, the feasibility tolerance for constraints of LCQP, QCQP, and SDP is set to $1 \times 10^{-6}$ and the tolerance for the stopping criteria is set to $1 \times 10^{-6}$.

\subsection{Synthetic scenes}

In this section, we show some experiments on synthetic dataset [15]. The dataset consists of ten objects, which have a smooth shape, and the dataset contains ideally complete 3D shape data. There is another dataset for evaluating the shape-from-shading or photometric stereo method $([9,33])$, but [15] is designed for synthetic evaluation and suits our evaluation. We show the results of five objects among them, labeled "blob01" to "blob05," rendered under a directional light source $\mathbf{l}=(0,0,1)^{\top}$. Figure 2 summarizes the results of various settings: (a) ORIGINAL setting with the Lagrangian relaxation, (b) INSIDE relaxation, (c) BOX relaxation, (d) OPEN relaxation, (e) PIECEWISE solution method described in Section 3.5, and (f) ITERATIVE method of [10]. For each scene, top row shows the estimated 

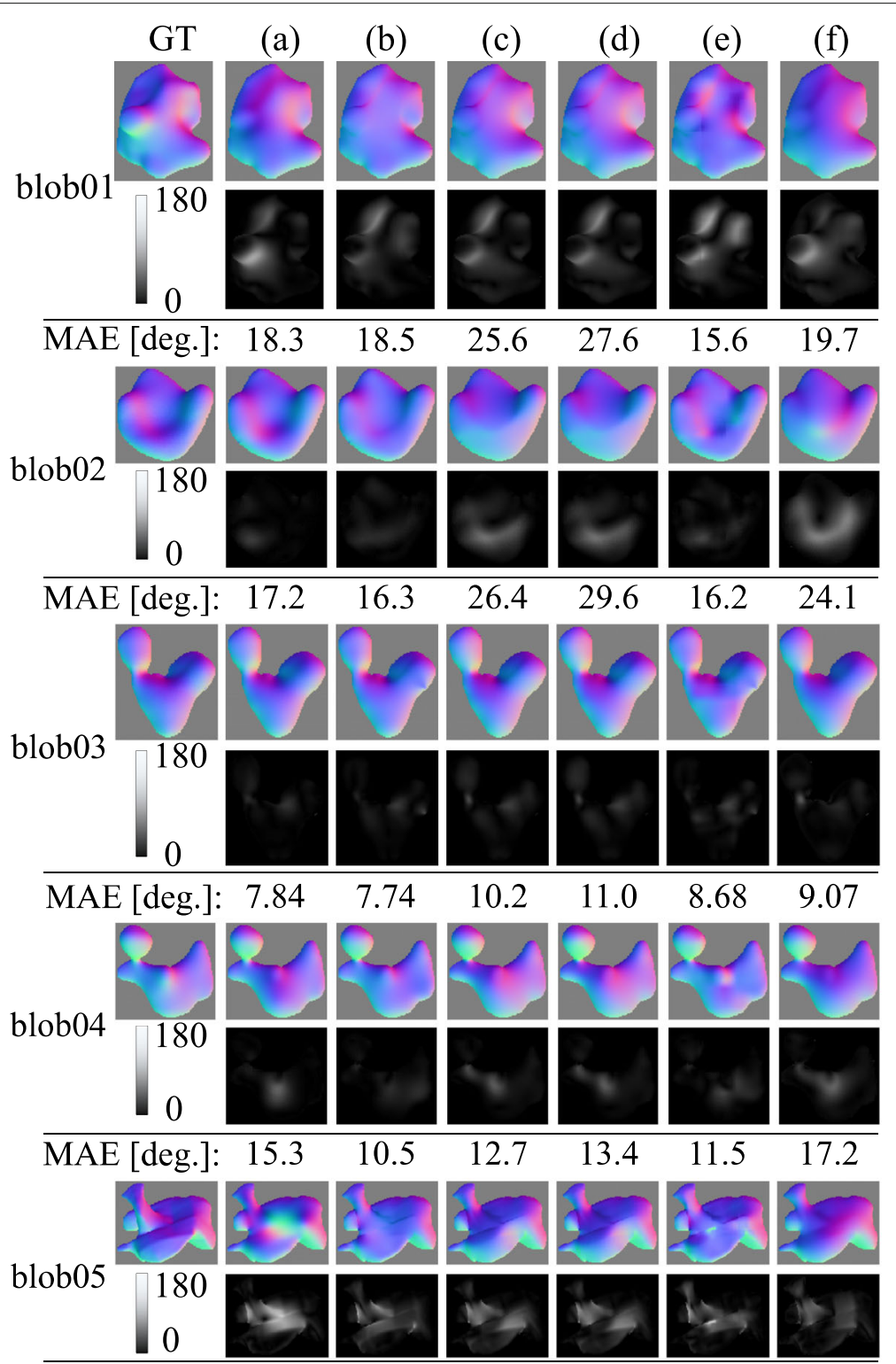

$13.4 \quad 11.5$

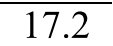

MAE [deg.]: 22.0

22.2

24.2
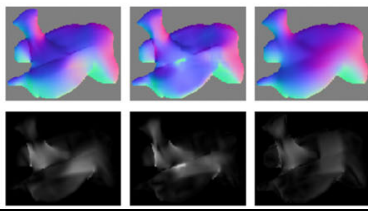

Fig. 2 Results on Blobby dataset [15]. From left to right, surface normal and angular error maps are shown for (a) ORIGINAL, (b) INSIDE, (c) BOX, (d) OPEN, (e) PIECEWISE, and (f) ITERATIVE methods. GT indicates the ground truth normal maps, and the values represent corresponding MAEs

surface normal, and the bottom row depicts the angular error map and corresponding mean angular error (MAE). For the ORIGINAL method with Lagrangian relaxation, we carefully picked the weight parameters $\left(\lambda_{1}, \lambda_{2}, \lambda_{3}\right)=(512,2048,32)$ with numerical simulation based on ground truth. It shows that aside from the ORIGINAL method, the INSIDE relaxation tends to yield favorable result compared to BOX and OPEN relaxations. The trend is inherited in the PIECEWISE method that uses the INSIDE relaxation in a sequential manner. The ITERATIVE method also shows higher accuracy compared to BOX and OPEN relaxations. The ORIGINAL setting shows the highest accuracy in two scenes, but the weight (hyper) parameters of the Lagrangian relaxation have been carefully chosen for producing the results.

Discussion on Lagrangian relaxation for ORIGINAL. The Lagrangian relaxation of the ORIGINAL setting has two obvious issues. One is the non-convexity of the problem, which implies that the solution may depend on the initial guess. The other is that the hyper parameters $\lambda_{1}, \lambda_{2}$, and $\lambda_{3}$ of [8] need to be properly chosen for expecting accurate estimates; however, unfortunately, 

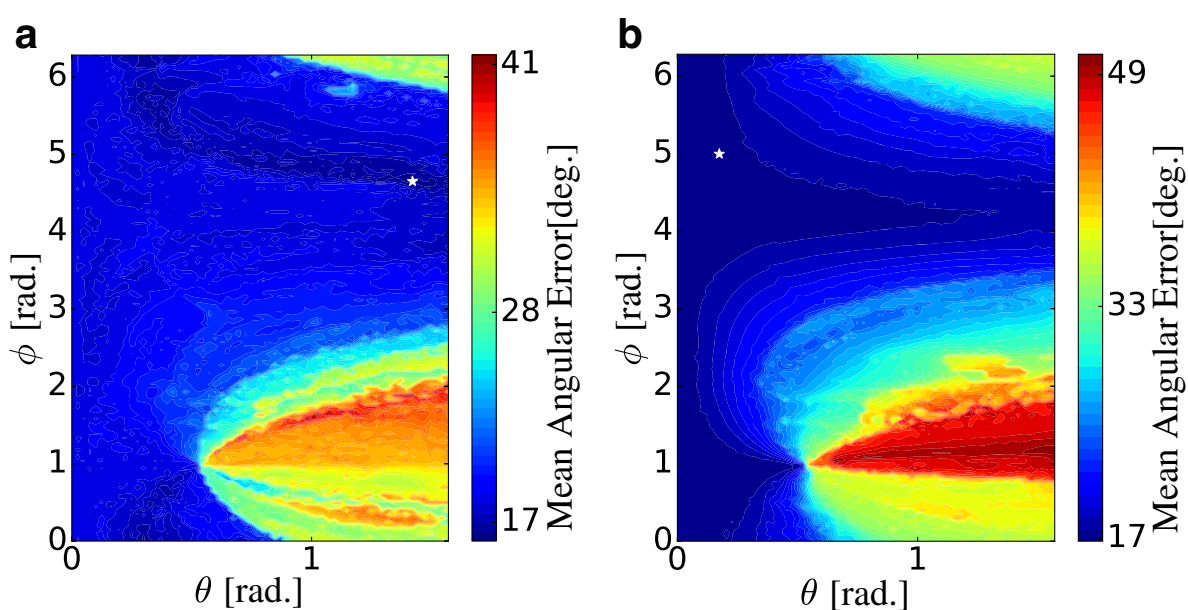

Fig. 3 Variation of mean angular errors (MAEs) with respect to the initial guess of surface normal using the Lagrangian relaxation of the ORIGINAL setting for blob01 (left) and blob02 (right) scenes. The initial guess of normal vectors are uniformly sampled from the hemisphere in the spherical coordinates $(\theta, \phi)$. The star markers in the figure represent the best initial guesses

the optimal hyper parameters are generally unknown and scene-dependent.

Figure 3 shows the plot of MAEs that are obtained by changing the initial guess of the surface normal for the blob01 and blob02 scenes using the Lagrangian relaxation of the ORIGINAL setting. In the figures, $x$ - and $y$-axes correspond to the azimuth $\theta$ and polar $\phi$ angles of the initial guess of the surface normal. The MAE drastically varies with the small variations of initial guess for the surface normal, and the variation has dependency on the scene.

To see the effect of the choice of hyper parameters, we altered the hyper parameters $\lambda_{1}, \lambda_{2}$, and $\lambda_{3}$ of [8] and observed the resulting MAEs. One of the results using the blob03 scene is shown in Fig. 4, in which the hyper parameters are set to $\lambda_{1}=\lambda_{2}=\lambda_{3} \in\{1,10,100,1000,10000\}$. The MAE varies significantly depending on the choice of the parameters, and it illustrates the difficulty of applying the Lagrangian relaxation of the ORIGINAL problem.

Comparison to existing methods. We compared our method with P-SFS and XIONG. While our method requires the boundary conditions to work properly, in order to compare with the P-SFS and XIONG methods that do not require them, we eliminate the boundary condition from the INSIDE relaxation. As a result, there remains a rotation ambiguity in the solution. Therefore, we applied rotation alignment of the estimated normal map for the purpose of comparison. We determine the rotation matrix $\mathbf{R} \in \mathbb{R}^{3 \times 3}$ by solving the following problem:

$$
\begin{array}{ll}
\underset{\mathbf{R}}{\operatorname{minimize}} & \left\|\mathbf{N}^{*}-\mathbf{R} \hat{\mathbf{N}}\right\|_{F}^{2} \\
\text { subject to } & \mathbf{R} \mathbf{R}^{\top}=\mathbf{I},
\end{array}
$$

where $\mathbf{N}^{*}$ and $\hat{\mathbf{N}}$ are the ground truth and estimated normal maps, respectively. This problem is known as the orthogonal Procrustes problem [12], and the solution method is proposed in [32]. XIONG directly estimates the depth rather than surface normal; therefore, to compare with other methods in the space of surface normal, we compute the normal map from the estimated depth map. Figure 5 shows one of the representative results. From left to right, it shows the ground truth normal map, (a) result of the INSIDE relaxation with boundary conditions, (b) INSIDE relaxation without boundary conditions, (c) PSFS method , and (d) XIONG method. "(b) - aligned" and "(c) - aligned" are the rotation aligned results of (b) and (c). While the result of (a) is convincing, (b) and (c) are rather far from the ground truth due to that the surface normals are not anchored by boundary conditions, containing the rotation ambiguity. Also, compared with (d), (a) achieves the better estimation.

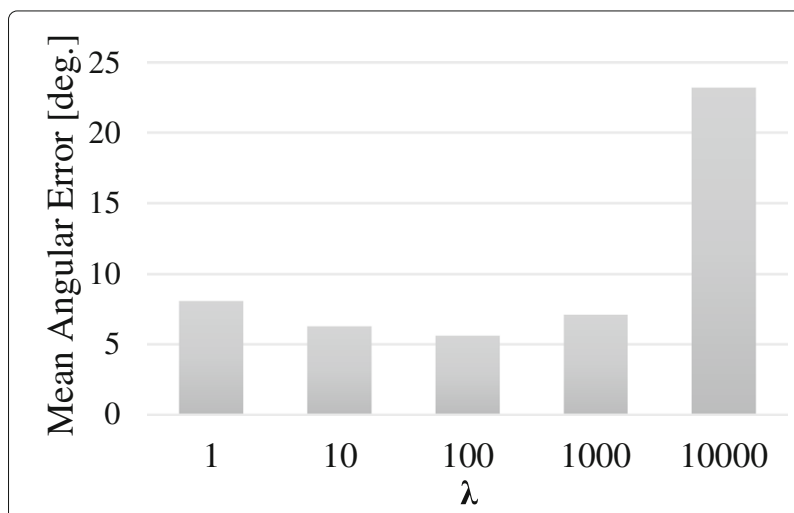

Fig. 4 Variation of mean angular errors (MAEs) with respect to the hyper parameters $\lambda_{1}, \lambda_{2}$, and $\lambda_{3}$ for blob03 scene. $\lambda_{1}=\lambda_{2}=\lambda_{3}=\lambda$, where $\lambda \in\{1,10,100,1000,10000\}$ 


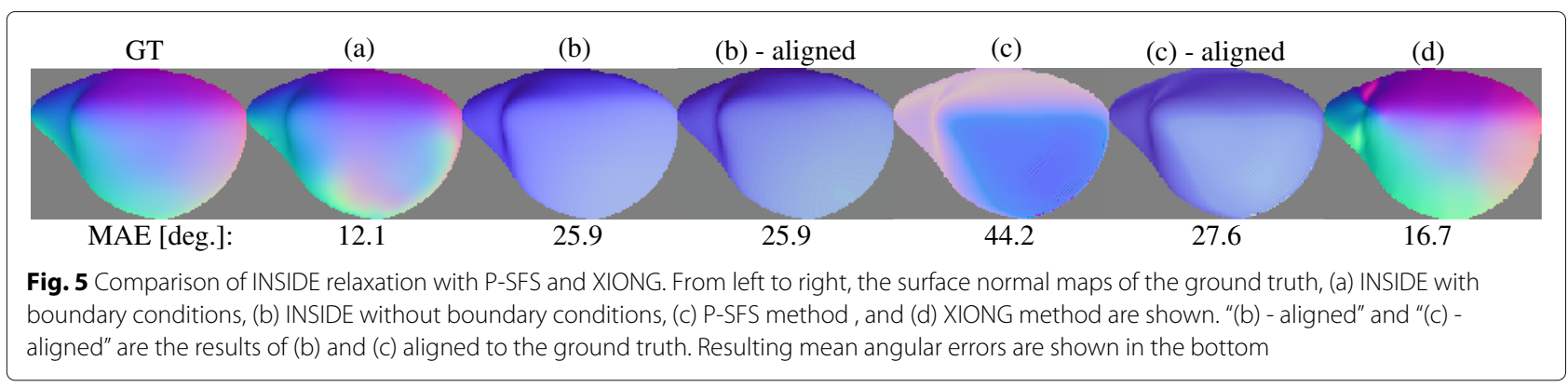

Speed and accuracy. Figure 6 summarizes the computation times and accuracies of various methods applied to blob01-blob05 datasets. The $x$ - and $y$-axes represent the log-scale processing time and MAE respectively. The mean scores of MAEs are plotted by circle, and their minimum and maximum time/accuracy are indicated by the associated bars. It can be seen that the PIECEWISE method significantly reduces the computation time compared to the INSIDE relaxation with retaining the accuracy. OPEN and BOX relaxations are faster; however, they suffer from inaccuracy due to the loose relaxation. The ORIGINAL method with Lagrangian relaxation shows a good trade-off as we have carefully selected a good set of hyper parameters. The MAE may significantly vary depending on the selection of hyper parameters as discussed earlier. The ITERATIVE method is the most efficient one among them, while MAEs were consistently larger than PIECEWISE, INSIDE, and ORIGINAL methods.

\subsection{Real-world data}

Real-world data contains observations that deviate from the assumed image formation model. Namely, there are two major factors: non-uniform diffuse albedos and non-Lambertian surface reflectances. Due to these unmodelled errors, the brightness [2] and boundary [4] constraints can conflict, resulting in no feasible solutions.
For the real-world data experiment, we therefore relax these hard constraints as soft ones as:

INSIDE relaxation:

$\begin{array}{ll}\underset{\mathbf{N}}{\operatorname{minimize}} & \frac{1}{2}\|\mathbf{N D}\|_{F}^{2}+\lambda_{1}\|\mathbf{N F}-\mathbf{G}\|_{F}^{2}+\lambda_{2}\left\|\mathbf{I}^{\top} \mathbf{N}-\mathbf{m}^{\top}\right\|_{2}^{2} \\ \text { subject to } & \left\|\mathbf{n}_{i}\right\|_{2}^{2} \leq 1, \quad 0 \leq n_{i z}, \quad \forall i \in\{1 \ldots p\} .\end{array}$

BOX relaxation:

$\underset{\mathbf{N}}{\operatorname{minimize}} \frac{1}{2}\|\mathbf{N D}\|_{F}^{2}+\lambda_{1}\|\mathbf{N F}-\mathbf{G}\|_{F}^{2}+\lambda_{2}\left\|\mathbf{I}^{\top} \mathbf{N}-\mathbf{m}^{\top}\right\|_{2}^{2}$ subject to $\quad-1 \leq n_{i x}, n_{i y} \leq 1,0 \leq n_{i z} \leq 1, \forall i \in\{1, \ldots, p\}$.

The results are summarized in Fig. 7. In the figure, "cat" data is from DiLiGenT [33] dataset, in which the ground truth is taken by the laser sensor. We picked up "cat" in DiLiGenT because it is the most Lambertian-like object. For other data, we have obtained the ground truth by a conventional least-squares photometric stereo [39] using 16 light sources. We selected these four objects: "wall-paper," "coin," and "logo," which have diffuse surfaces. From left to right, it shows the estimated surface normal and angular error maps of (a) ORIGINAL with Lagrangian relaxation, (b) INSIDE, (c) BOX, (d) OPEN, (e) PIECEWISE, and (f) ITERATIVE methods. Although the surface details are smoothed out due to the smoothness constraint, overall structures can be better observed by properly accounting for the unit norm

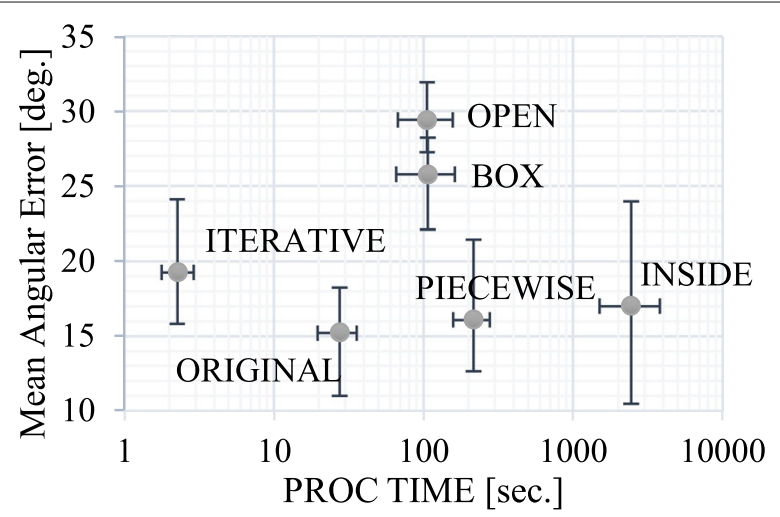

Fig. 6 Computation time ( $x$-axis) and mean angular error ( $y$-axis) for various settings assessed using blob01-blob05 


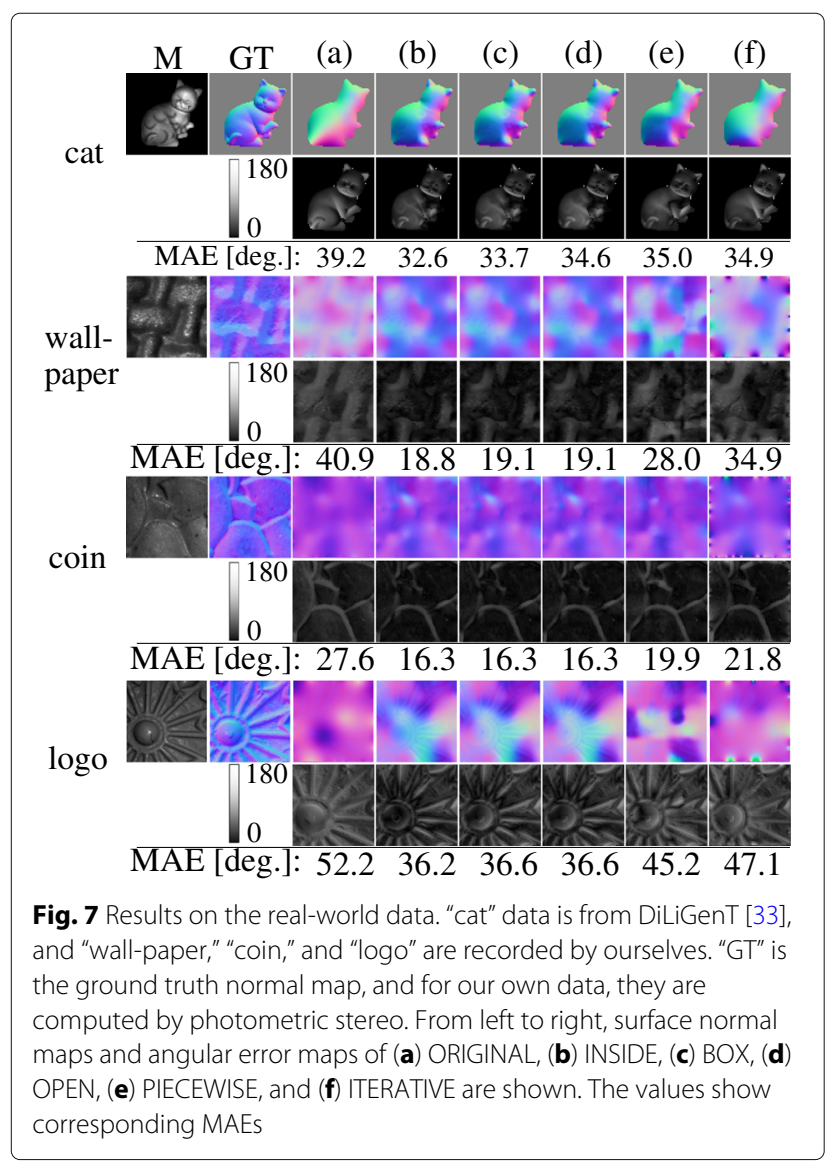

constraint with a tight relaxation by (b) compared to the result of (a) and (f). The PIECEWISE method in (e) also yields lower accuracy as well in this case but still producing results closer to the ground truth compared to (a) and (f).

Discussions on Lagrangian relaxation for the realworld data We examine Lagrangian relaxations of INSIDE, BOX, and OPEN methods using the real-world data for assessing their capabilities of handling unmodelled errors. The formulations are all convex problems; therefore, the solution does not depend on the initial guess. Here, we discuss the effect of the choice of hyper parameters $\lambda_{1}$ and $\lambda_{2}$.

We alter the hyper parameters $\lambda_{1}$ and $\lambda_{2}$ and observe the resulting mean angular errors (MAEs) . The results using the "cat," "wall-paper," "coin," and "logo" scenes are summarized in Fig. 8, in which the hyper parameters are set to $\lambda_{1}=\lambda_{2}=\lambda \in\{1,100,10000\}$.

While this result shows that the choice of hyper parameters has little effect on overall MAEs, it still locally affects surface normal estimates. For example, errors near ear and forefoot of "cat" are decreased with large hyper parameters in Fig. 9. Because the areas of ear and forefoot are not smooth, surface normal can be correctly estimated by emphasizing on the brightness and occluding boundary constraints rather than the smoothness constraint.

\section{Discussion}

This paper studied the unit norm constraint that appears in general shape-from-shading problems. We showed various convex relaxation strategies for the unit norm constraint, as well as a non-convex relaxation of the original problem using a Lagrangian relaxation. It has been shown that the INSIDE relaxation, which gives a tight convex surrogate for the original unit norm constraint, yields favorable results, and we developed a piecewise solution method for accelerating the shape estimation.

It has been shown that with carefully selected hyperparameters, Lagrangian relaxation works well in terms of its speed and accuracy. However, unfortunately, such a priori knowledge is generally unavailable in realworld situations. For shape-from-shading to work with real-world applications, the INSIDE relaxation appears to be a favorable option when dealing with the unit norm constraint. With advanced convex optimization
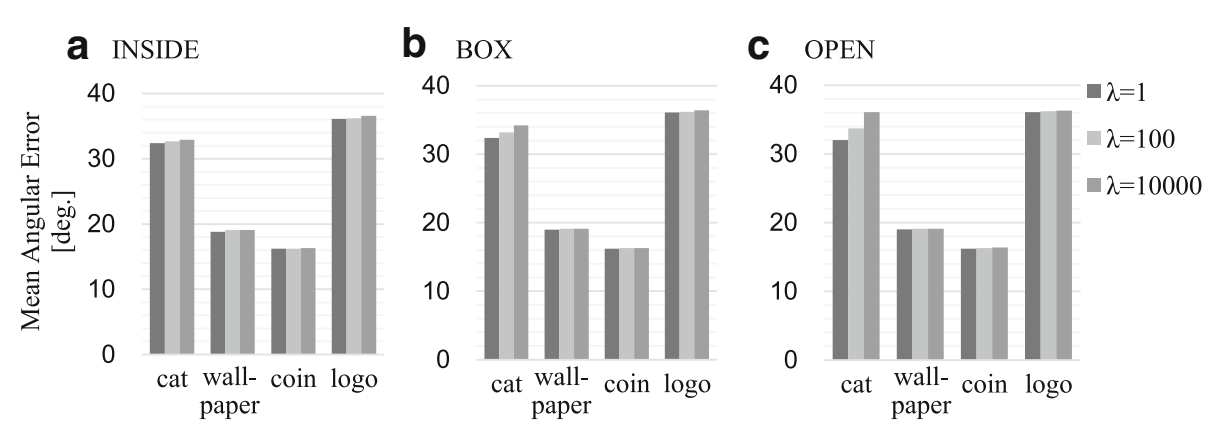

Fig. 8 Variation of mean angular errors (MAEs) with respect to the hyper parameters $\lambda_{1}$ and $\lambda_{2} \cdot \lambda_{1}=\lambda_{2}=\lambda, \in[1,100,10000]$. The MAEs of a INSIDE, b BOX, and c OPEN for four scenes ("cat," "wall-paper," "coin," and "logo") are shown 


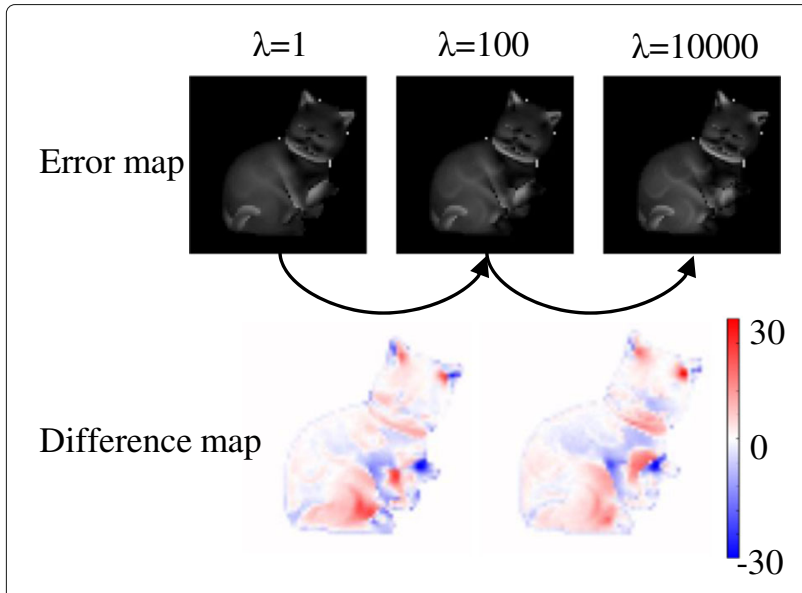

Fig. 9 Error map and difference map of INSIDE method for "cat" scene

techniques and mature linear algebra packages, the computation of shape-from-shading is made significantly more efficient. We are interested in fusing this basic study into other recent works that use other prior knowledge for making shape-from-shading further applicable.

As a practical issue, the proposed method needs the annotation of the occluding boundary. In a controlled setting, this annotation could be semi-automated by sophisticated segmentation tools, such as $[10,14,21]$, and we consider that this information is somewhat accessible in practice as various previous shape-from-shading works assumed.

\section{Endnotes}

${ }^{1}$ The non-convexity of the problem discussed in this paper is different from the concave/convex ambiguity that inherently appears in shape-from-shading problems.

${ }^{2}$ Gurobi Optimizer: http://www.gurobi.com/products/ gurobi-optimizer

${ }^{3} \mathrm{http} / /$ vision.seas.harvard.edu/qsfs/

\section{Appendix 1. Lagrange relaxation subproblem}

Search direction $\mathbf{d}^{(k)}$ for the Levenberg-Marquardt algorithm is determined by solving the following subproblem:

$$
\begin{array}{ll}
\text { minimize } & \left\|f\left(\mathbf{x}^{(k)}\right)+f^{\prime}\left(\mathbf{x}^{(k)}\right) \mathbf{d}^{(k)}\right\|_{2}^{2}+\kappa_{k}\left\|\mathbf{d}^{(k)}\right\|_{2}^{2} \\
\text { subject to } & 0 \leq n_{i} z .
\end{array}
$$

A positive parameter $\kappa_{k}$ is used to control regularization by $\left\|\mathbf{d}^{(k)}\right\|_{2}^{2} \cdot f\left(\mathbf{x}^{(k)}\right)$ and $f^{\prime}\left(\mathbf{x}^{(k)}\right)$ are given by

$$
\begin{aligned}
& f(\mathbf{x})=\left[\begin{array}{c}
\frac{1}{\sqrt{2}}\left\|\mathbf{D}_{\otimes} \mathbf{x}\right\|_{2} \\
\sqrt{\lambda_{1}}\left\|\mathbf{L}_{\otimes} \mathbf{x}-\mathbf{m}^{\top}\right\|_{2} \\
\sqrt{\lambda_{2}}\left\|\mathbf{F}_{\otimes} \mathbf{x}-\mathbf{g}\right\|_{2} \\
\sqrt{\lambda_{3}}\left(\left\|\mathbf{n}_{1}\right\|_{2}^{2}-1\right) \\
\vdots \\
\sqrt{\lambda_{3}}\left(\left\|\mathbf{n}_{p}\right\|_{2}^{2}-1\right)
\end{array}\right] \in \mathbb{R}^{p+3}
\end{aligned}
$$

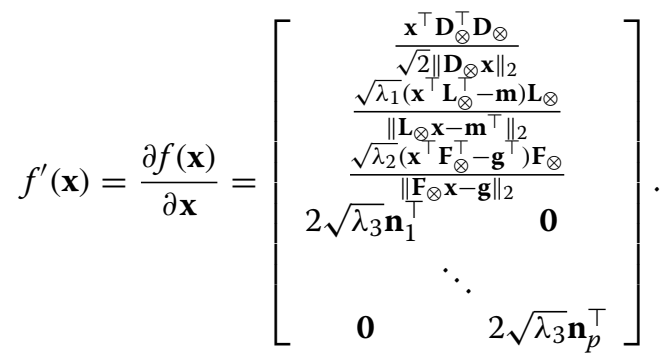

The subproblem [12] is a convex quadratic programming problem and thus has a unique solution for $\mathbf{d}^{(k)}$.

\section{Appendix 2: SOCP for INSIDE relaxation}

The SOCP minimizes $u$ for the upper bound of $\frac{1}{2}\left\|\mathbf{D}_{\otimes} \mathbf{x}\right\|_{2}^{2}$ as

$$
\begin{array}{cl}
\underset{u, \mathbf{x}}{\operatorname{minimize}} & u \\
\text { subject to } & \mathbf{L}_{\otimes} \mathbf{x}-\mathbf{m}^{\top}=\mathbf{0}, \\
& \mathbf{F}_{\otimes} \mathbf{x}-\mathbf{g}=\mathbf{0}, \\
& 0 \leq \mathbf{s}_{i}^{\top} \mathbf{x}, \quad(i=3,6, \ldots, 3 p), \\
& \left\|\left[\frac{1}{2} u-1 \mathbf{x}^{\top} \mathbf{D}_{\otimes}\right]^{\top}\right\|_{2}^{2} \leq \frac{1}{2} u+1, \\
& \left\|\left[-1 \mathbf{x}^{\top} \mathbf{K}_{i}\right]^{\top}\right\|_{2}^{2} \leq 1, \quad(i=1, \ldots, p) .
\end{array}
$$

SOCP can be efficiently solved by a primal-dual interiorpoint method, which solves the following modified KKT (Karush-Kuhn-Tucker) conditions [17, 22] with letting y denote $\mathbf{y}=\left[u, \mathbf{x}^{\top}\right]^{\top}$ :

$$
r_{t}(\mathbf{y}, \mu, v)=\left[\begin{array}{c}
\nabla_{\mathbf{y}} u+(\mathcal{D} H(\mathbf{y}))^{\top} \mu+\mathbf{A}^{\top} v \\
-\operatorname{diag}(\mu) H(\mathbf{y})-\frac{1}{t} \mathbf{1} \\
\mathbf{A y}-\mathbf{b}
\end{array}\right]=\mathbf{0},
$$

where $\mu \in \mathbb{R}^{2 p+1}$ and $\nu \in \mathbb{R}^{6 p}$ are Lagrange multipliers and $t$ is a parameter to control approximation in the barrier method. Parameters $\nabla_{\mathbf{y}} u, H(\mathbf{y}), \mathcal{D} H(\mathbf{y}), \mathbf{A}$, and $\mathbf{b}$ are given by 


$$
\begin{aligned}
& \nabla_{\mathbf{y}} u=\left[\begin{array}{llll}
1 & 0 & \ldots & 0
\end{array}\right]^{\top}
\end{aligned}
$$

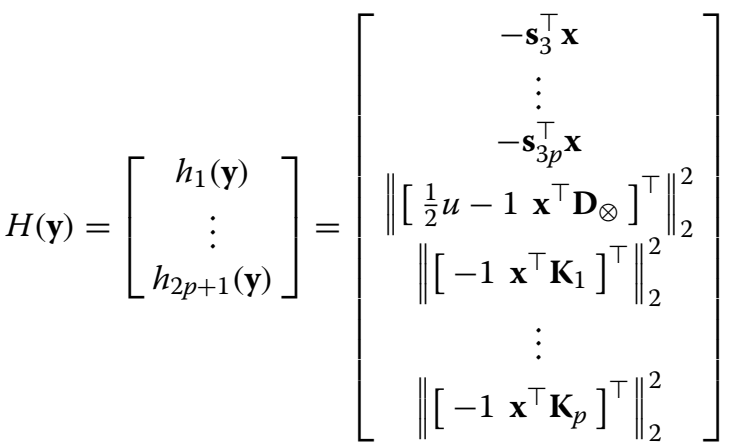

$$
\begin{aligned}
& \mathcal{D} H(\mathbf{y})=\left[\begin{array}{c}
\nabla_{\mathbf{y}} h_{1}^{\top}(\mathbf{y}) \\
\vdots \\
\nabla_{\mathbf{y}} h_{2 p+1}^{\top}(\mathbf{y})
\end{array}\right]=\left[\begin{array}{c}
0-\mathbf{s}_{3}^{\top} \\
\vdots \\
0-\mathbf{s}_{3 p}^{\top} \\
2 \mathbf{y}^{\top} \mathbf{P}^{\top} \mathbf{P}+2 \mathbf{c}^{\top} \mathbf{P} \\
2 \mathbf{y}^{\top} \mathbf{Q}_{1}^{\top} \mathbf{Q}_{1}+2 \mathbf{c}^{\top} \mathbf{Q}_{1} \\
\vdots \\
2 \mathbf{y}^{\top} \mathbf{Q}_{p}^{\top} \mathbf{Q}_{p}+2 \mathbf{c}^{\top} \mathbf{Q}_{p}
\end{array}\right] \\
& \mathbf{A}=\left[\begin{array}{ll}
\mathbf{0} & \mathbf{L}_{\otimes} \\
\mathbf{0} & \mathbf{F}_{\otimes}
\end{array}\right], \quad \text { and } \mathbf{b}=\left[\begin{array}{c}
\mathbf{m}^{\top} \\
\mathbf{g}
\end{array}\right] \text {, }
\end{aligned}
$$

where

$$
\begin{aligned}
& \mathbf{P}=\left[\begin{array}{cc}
1 / 2 & \mathbf{0} \\
\mathbf{0} & \mathbf{D}_{\otimes}
\end{array}\right], \quad \mathbf{Q}_{i}=\left[\begin{array}{cc}
0 & \mathbf{0} \\
\mathbf{0} & \mathbf{K}_{i}
\end{array}\right], \\
& \mathbf{c}=\left[\begin{array}{llll}
-1 & 0 & \ldots & 0
\end{array}\right]^{\top}\left(\in \mathbb{R}^{3 p+1}\right) .
\end{aligned}
$$

The modified KKT equations can be solved by Newton's method that changes $\mathbf{y}, \mu$, and $v$ by Newton steps $\Delta \mathbf{y}, \Delta \mu$, and $\Delta v$. The newton step is characterized by the following linear equations $[4]$

$r_{t}(\mathbf{y}+\Delta \mathbf{y}, \mu+\Delta \mu, v+\Delta v) \sim \mathcal{D} r_{t}(\mathbf{y}, \mu, v)[\Delta \mathbf{y}, \Delta \mu, \Delta v]^{\top}$,

which results in a system of linear equations

$$
\left[\begin{array}{ccc}
\sum_{i=1}^{2 p+1} \mu_{i} \nabla^{2} h_{i}(\mathbf{y}) & \mathcal{D} H^{\top}(\mathbf{y}) & \mathbf{A}^{\top} \\
-\operatorname{diag}(\lambda) \mathcal{D} H^{\top}(\mathbf{y}) & -\operatorname{diag}(H(\mathbf{y})) & \mathbf{0} \\
\mathbf{A} & \mathbf{0} & \mathbf{0}
\end{array}\right]\left[\begin{array}{c}
\Delta \mathbf{y} \\
\Delta \mu \\
\Delta v
\end{array}\right]=-\left[\begin{array}{c}
r_{\text {dual }} \\
r_{\text {cent }} \\
r_{\text {pri }}
\end{array}\right],
$$

where $r_{\text {dual }}, r_{\text {cent }}$, and $r_{\text {pri }}$ are residuals that are evaluated on the first, second, and third row block matrices in the KKT Eq. (13), respectively, after the previous Newton step.

\section{Appendix 3: KKT for Box relaxation}

The KKT conditions for the BOX relaxation case are:

$$
r_{t}(\mathbf{y}, \mu, v)=\left[\begin{array}{c}
\frac{1}{2} \nabla_{\mathbf{x}}\left\|\mathbf{D}_{\otimes} \mathbf{x}\right\|_{2}^{2}+(\mathcal{D} E(\mathbf{x}))^{\top} \mu+\mathbf{C}^{\top} v \\
-\operatorname{diag}(\mu) E(\mathbf{x})-\frac{1}{t} \mathbf{1} \\
\mathbf{C x}-\mathbf{b}
\end{array}\right]=\mathbf{0},
$$

where

$$
\begin{gathered}
E(\mathbf{x})=\left[\begin{array}{c}
e_{1}(\mathbf{x}) \\
\vdots \\
e_{6 p}(\mathbf{x})
\end{array}\right]=\left[\begin{array}{c}
-\mathbf{s}_{1}^{\top} \mathbf{x}-1 \\
-\mathbf{s}_{2}^{\top} \mathbf{x}-1 \\
-\mathbf{s}_{3}^{\top} \mathbf{x} \\
\vdots \\
-\mathbf{s}_{3 p}^{\top} \mathbf{x} \\
\mathbf{s}_{1}^{\top} \mathbf{x}-1 \\
\vdots \\
\mathbf{s}_{3 p}^{\top} \mathbf{x}-1
\end{array}\right], \\
\mathcal{D} E(\mathbf{x})=\left[\begin{array}{c}
-\mathbf{s}_{1}^{\top} \\
\vdots \\
-\mathbf{s}_{3 p}^{\top} \\
\mathbf{s}_{1}^{\top} \\
\vdots \\
\mathbf{s}_{3 p}^{\top}
\end{array}\right], \quad \mathbf{C}=\left[\begin{array}{c}
\mathbf{L}_{\otimes} \\
\mathbf{F}_{\otimes}
\end{array}\right] .
\end{gathered}
$$

The Newton steps $\Delta \mathbf{y}, \Delta \mu$, and $\Delta v$ are derived by solving the following equations

$$
\left[\begin{array}{ccc}
\mathbf{D}_{\otimes}^{2}+\sum_{i=1}^{6 p} \mu_{i} \nabla^{2} e_{i}(\mathbf{x}) & \mathcal{D} E^{\top}(\mathbf{x}) & \mathbf{C}^{\top} \\
-\operatorname{diag}(\lambda) \mathcal{D} E^{\top}(\mathbf{x}) & -\operatorname{diag}(E(\mathbf{x})) & \mathbf{0} \\
\mathbf{C} & \mathbf{0} & \mathbf{0}
\end{array}\right]\left[\begin{array}{c}
\Delta \mathbf{y} \\
\Delta \mu \\
\Delta v
\end{array}\right]=-\left[\begin{array}{c}
r_{\text {dual }} \\
r_{\text {cent }} \\
r_{\text {pri }}
\end{array}\right],
$$

where $\mathbf{D}_{\otimes}^{2}=D_{\otimes}^{\top} D_{\otimes}$, and $r_{\text {dual }}, r_{\text {cent }}$, and $r_{p r i}$ are residuals that are respectively evaluated on the first, second, and third row block matrices in the modified KKT Eq. (14) after the previous Newton step.

\section{Funding}

This work was supported by JSPS Grant-in-Aid for Scientific Research (A) (KAKENHI), grant number JP16H01732. The funders had no role in the study design, data collection and analysis, decision to publish, or preparation of the manuscript.

\section{Availability of data and materials}

The datasets generated during and/or analyzed during the current study are available from the corresponding author on reasonable request.

\section{Authors' contributions}

HS carried out derivation of the solution methods, implementation, data acquisition, and experimentation, and drafted the manuscript. MS participated in the design of the convex optimization techniques described in the manuscript, contributed to developing solution methods, and helped to draft the manuscript. MT participated in the sequence alignment. YM conceived of the study, participated in its design and coordination, and helped to draft the manuscript. All authors read and approved the final manuscript.

\section{Competing interests}

The authors declare that they have no competing interests.

\section{Publisher's Note}

Springer Nature remains neutral with regard to jurisdictional claims in published maps and institutional affiliations.

Received: 27 April 2017 Accepted: 23 May 2018

Published online: 14 June 2018 


\section{References}

1. Anstreicher KM (2009) Semidefinite programming versus the reformulation-linearization technique for nonconvex quadratically constrained quadratic programming. J Glob Optim 43(2-3):471-484

2. Barron JT, Malik J (2011) High-frequency shape and albedo from shading using natural image statistics. In: Proceedings of IEEE Conference on Computer Vision and Pattern Recognition (CVPR). IEEE, New Jersey. pp 2521-2528

3. Blanz V, Vetter $T$ (2003) Face recognition based on fitting a $3 d$ morphable model. IEEE Trans Pattern Anal Mach Intell 25(9):1063-1074

4. Boyd S, Vandenberghe L (2004) Convex optimization. Cambridge University Press, New York

5. Brooks MJ, Horn BKP (1985) Shape and source from shading. In: Proceedings of International Joint Conference on Artificial Intelligence. Association for the Advancement of Artificial Intelligence, California. pp 932-936

6. Durou J-D, Falcone M, Sagona M (2008) Numerical methods for shape-from-shading: a new survey with benchmarks. Comp Vision Image Underst 109(1):22-43

7. Ecker A, Jepson AD (2010) Polynomial shape from shading. In: Proceedings of IEEE Conference on Computer Vision and Pattern Recognition (CVPR). IEEE, New Jersey. pp 145-152

8. Forsyth DA (2011) Variable-source shading analysis. Int J Comput Vis 91(3):280-302

9. Frankot RT, Chellappa R (1988) A method for enforcing integrability in shape from shading algorithms. PAMI 10(4):439-451

10. Geman D, Geman S, Graffigne C, Dong P (1990) Boundary detection by constrained optimization. IEEE Trans Pattern Anal Mach Intell 12(7):609-628

11. Horn BKP (1970) Shape from shading: a method for obtaining the shape of a smooth opaque object from one view. Technical Report AITR-232. MIT

12. Hurley JR, Cattell RB (1962) The procrustes program: producing direct rotation to test a hypothesized factor structure. Syst Res Behav Sci $7(2): 258-262$

13. Ikeuchi K, Horn BKP (1981) Numerical shape from shading and occluding boundaries. Artif Intell 17(1-3):141-184

14. Isola P, Zoran D, Krishnan D, Adelson EH (2014) Crisp boundary detection using pointwise mutual information. Springer, Switzerland

15. Johnson MK, Adelson EH (2011) Shape estimation in natural illumination. In: Proceedings of IEEE Conference on Computer Vision and Pattern Recognition (CVPR). IEEE, New Jersey. pp 2553-2560

16. Kanzow C, Yamashita N, Fukushima M (2004) Levenberg-Marquardt methods with strong local convergence properties for solving nonlinear equations with convex constraints. J Comput Appl Math 172(2):375-397

17. Karush W (1939) Minima of Functions of Several Variables with Inequalities as Side Conditions. Master's thesis, Department of Mathematics. University of Chicago, Chicago, IL

18. Khan N, Tran L, Tappen M (2009) Training many-parameter shape-from-shading models using a surface database. In: Computer Vision Workshops (ICCV Workshops. IEEE, New Jersey

19. Kimmel R, Bruckstein AM (1992) Shape from shading via level sets. Israel Institute of Technology. Technical Report CIS Report

20. Kimmel R, Sethian JA (2001) Optimal algorithm for shape from shading and path planning. J Math Imaging Vision 14(3):237-244

21. Kokkinos I (2010) Highly accurate boundary detection and grouping. In: Proceedings of IEEE Conference on Computer Vision and Pattern Recognition, CVPR. IEEE, New Jersey. pp 2520-2527

22. Kuhn HW Tucker AW (1951) Nonlinear programming. In: Proceedings of 2nd Berkeley Symposium. University of California Press, Berkeley. pp 481-492

23. Lee $\mathrm{CH}$, Rosenfeld A (1985) Improved methods of estimating shape from shading using the light source coordinate system. Artif Intell 26(2):125-143

24. Levenberg K (1944) A method for the solution of certain non-linear problems in least squares. Q Appl Math 2(2):164-168

25. Lobo MS, Vandenberghe L, Boyd S, Lebret H (1998) Applications of second-order cone programming. Linear Algebra Appl 284(1):193-228

26. Marquardt DW (1963) An algorithm for least-squares estimation of nonlinear parameters. J Soc Ind Appl Math 11(2):431-441

27. Pentland $A$ (1989) Shape information from shading: a theory about human perception. Spat Vis 4(2):165-182
28. Pentland AP (1982) Local shading analysis. Technical, Report Technica Note 272. SRI International

29. Queau Y, Melou J, Castan F, Cremers D, Durou J-D (2017) A variational approach to shape-from-shading under natural illumination. In: Energy Minimization Methods in Computer Vision and Pattern Recognition. Springer International Publishing, Switzerland

30. Richter SR, Roth S (2015) A discriminative approach to perspective shape from shading in uncalibrated illumination. Comput Graph 53:72-81

31. Richter SR, Roth S (2015) Discriminative shape from shading in uncalibrated illumination. In: Proceedings of IEEE Conference on Computer Vision and Pattern Recognition (CVPR). IEEE, New Jersey. pp 1128-1136

32. Schönemann PH (1966) A generalized solution of the orthogonal procrustes problem. Psychometrika 31(1):1-10

33. Shi B, Wu Z, Mo Z, Duan D, Yeung S-K, Tan P (2016) A benchmark dataset and evaluation for non-lambertian and uncalibrated photometric stereo. In: Proceedings of IEEE Conference on Computer Vision and Pattern Recognition (CVPR). IEEE, New Jersey

34. Smith WAP, Hancock ER (2002) Face recognition using shape-from-shading. In: Proceedings of British Machine Vision Conference (BMVC). The British Machine Vision Association and Society for Pattern Recognition, Durham. pp 1-10

35. Szeliski R (1990) Fast shape from shading. In: Faugeras O (ed). Proceedings of European Conference on Computer Vision (ECCV). IEEE, New Jersey. pp 359-368

36. Tankus A, Sochen N, Yeshurun Y (2005) Shape-from-shading under perspective projection. IJCV 63(1):21-43

37. Tsai PS, Shah M (1994) Shape from shading using linear approximation. Image Vis Comput J 12(8):487-498

38. Witkin AP (1983) Scale-space filtering. In: Proceedings of International Joint Conference on Artificial Intelligence. pp 1019-1021

39. Woodham RJ (1980) Photometric method for determining surface orientation from multiple images. Opt Engineerings 19(I):139-144

40. Wright SJ (1997) Primal-Dual Interior-Point Methods. Society for Industrial and Applied Mathematics, Philadelphia

41. Wu C, Wilburn B, Matsushita Y, Theobalt C (2011) High-quality shape from multi-view stereo and shading under general illumination. In: Proceedings of IEEE Conference on Computer Vision and Pattern Recognition (CVPR). IEEE, New Jersey. pp 969-976

42. Wu C, Narasimhan SG, Jaramaz B (2010) A multi-image shape-from-shading framework for near-lighting perspective endoscopes. Int J Comput Vis 86(2):211-228

43. Xiong Y, Chakrabarti A, Basri R, Gortler SJ, Jacobs DW, Zickler TE (2015) From shading to local shape. IEEE Trans Pattern Anal Mach Intell 37(1):67-79

44. Yu L-F, Yeung S-K, Tai Y-W, Lin S (2013) Shading-based shape refinement of rgb-d images. In: Proceedings of IEEE Conference on Computer Vision and Pattern Recognition (CVPR). IEEE, New Jersey. pp 1415-1422

45. Zhang R, Tsai P-S, Cryer JE, Shah M (1999) Shape from shading: A survey. IEEE Trans Pattern Anal Mach Intell 21(8):690-706

\section{Submit your manuscript to a SpringerOpen ${ }^{\circ}$ journal and benefit from:}

- Convenient online submission

- Rigorous peer review

- Open access: articles freely available online

- High visibility within the field

- Retaining the copyright to your article

Submit your next manuscript at $>$ springeropen.com 